\title{
Pierre Auger et la physique atomique
}

Jean-Pierre Briand (www.jpbriand.com)

Professeur émérite à l'Université Pierre et Marie Curie

\section{L'effet Auger, découvert}

en 1923, n'a guère suscité d'intérêt chez les physiciens

\section{atomistes (notamment en}

France) avant les années 70.

Près de cinquante ans de

désintérêt pour l'effet Auger

et son auteur (bien plus

connu à l'étranger qu'en

France). Pourquoi?

Jean-Pierre Briand, qui l'a

bien connu, nous explique ici

I'histoire assez extraordinaire

de cette découverte et

de la traversée du désert

qui l'a suivie : difficultés conceptuelles chez les physi-

ciens atomistes, cloisonnement entre cette collectivité et celle des physiciens nucléaires...

II nous présente également

quelques illustrations peu connues de la physique

«post-Auger».
Dans un précédent numéro de Reflets de la physique [1], Olivier Hardouin Duparc raconte l'histoire de la découverte de l'effet Auger et des controverses entre les physiciens de l'époque, comme Lise Meitner et Charles Ellis, sur l'origine des électrons observés lors des désintégrations nucléaires $\beta$. À l'époque de Pierre Auger on appelait indistinctement «électron $\beta$ » tout électron émis par un atome ou son noyau (on ne savait pas bien faire la différence). Pierre Auger n'était alors qu'un jeune thésard, un peu solitaire, qui s'intéressait à un processus purement atomique : la photo-ionisation, qu'il étudiait en utilisant une technique développée pour la physique nucléaire (la chambre de Wilson). Il avait découvert et parfaitement interprété un effet physique fondamental nouveau.

La découverte de l'effet Auger, en 1923, ne suscita, à l'époque, qu'un intérêt très modéré dans la collectivité des physiciens atomistes et ce processus, pourtant si fondamental, est resté étonnamment mal compris, voire ignoré, pratiquement pas enseigné, jusque dans les années 1965-70. Près de cinquante ans de désintérêt. Pourquoi ? L'histoire assez extraordinaire de l'effet Auger a été beaucoup plus marquée par le cloisonnement entre les différentes communautés scientifiques, ou l'évolution des technologies, que par des considérations scientifiques cohérentes, et ne s'est vraiment achevée que dans les années 70 .

\section{Les « électrons $\beta$ »}

Les physiciens nucléaires disposaient, pour étudier la radioactivité $\beta$, de spectrographes permettant de mesurer l'énergie cinétique des électrons par déflection de leurs trajectoires dans un champ magnétique. Ils observaient (fig. 1), surimposées aux spectres $\beta$ continus, des raies mono-énergétiques dont l'origine était mal comprise à l'époque, provenant, nous le savons maintenant, d'interactions de natures très différentes entre les noyaux radioactifs et le cortège électronique :

- la conversion interne qui se produit pendant une transition nucléaire. À l'issue d'une désintégration radioactive $\alpha$ ou $\beta$, le noyau final peut se trouver dans un état excité, qui est susceptible de se désexciter par émission d'un photon (émission $\gamma$ ). La conversion interne est un processus alternatif à l'émission $\gamma$, de cession directe de l'énergie d'excitation du noyau à son cortège électronique. Cette énergie est alors transférée à un électron du cortège de l'atome (de la couche $\mathrm{K}$ par exemple), qui est expulsé avec une énergie $\mathrm{T}$ égale à l'énergie $\mathrm{E}$ du niveau nucléaire moins l'énergie de liaison de cet électron : $\mathrm{T}=\mathrm{E}-\mathrm{B}_{\mathrm{K}}$;

- le réarrangement spontané, ultérieur, du cortège électronique de l'atome, lorsque celui-ci a été ionisé à l'issue d'une désintégration nucléaire, par exemple d'un

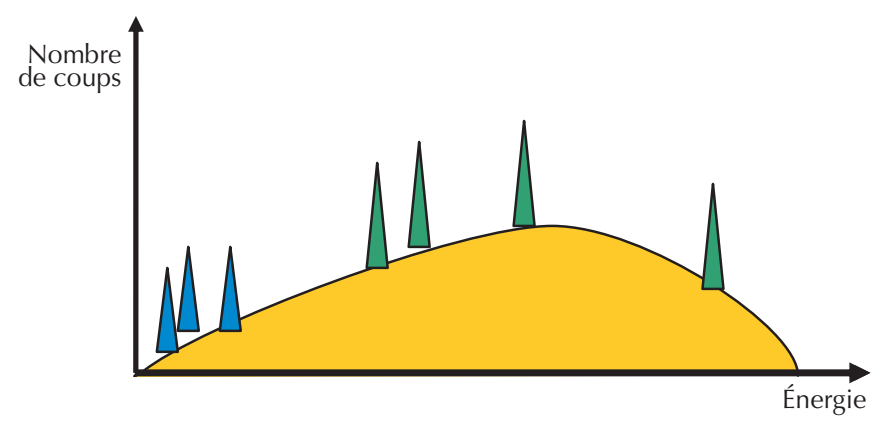

1. Spectre schématique d'électrons observé lors de désintégrations nucléaires $\beta$. En jaune : spectre continu $\beta$; en vert : raies de conversion interne (électrons « durs »); en bleu : transitions Auger (électrons « mous »). 


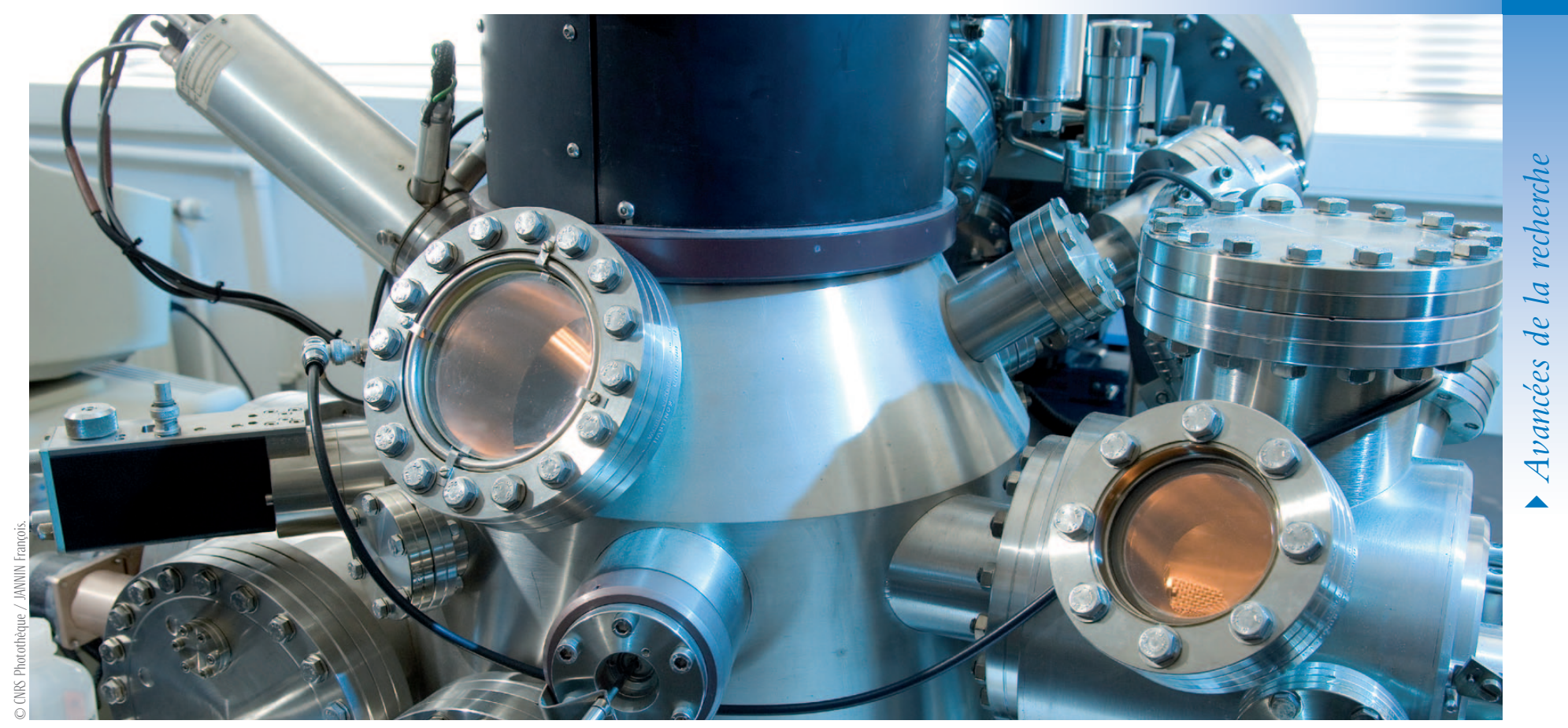

Microsonde à spectrométrie d'électrons Auger pour la caractérisation de matériaux avancés. L'excitation par un faisceau d'électrons d'une vingtaine de nm de diamètre permet d'obtenir une information chimique avec une résolution spatiale exceptionnelle.

processus de conversion interne. L'atome ionisé en couche profonde se désexcite alors par émission de rayons X ou... par éjection spontanée d'un de ses électrons : l'effet Auger (fig. 2b). Les énergies des raies observées ne dépendent alors que de la nature de l'atome formé.

On comprend aisément comment l'intrication de tous ces phénomènes mal connus ou mal compris ait pu amener à l'époque à de grandes confusions ou mésinterprétations. On comprend mal, par contre, pourquoi ces incompréhensions ont pu perdurer aussi longtemps (mésinterprétations encore malheureusement présentes dans un certain nombre d'ouvrages).

\section{L'effet Auger}

L'effet Auger est un effet physique conceptuellement simple : lorsque la répulsion entre les électrons d'un atome est plus forte que leur attraction par le noyau, l'atome n'est plus dans un état stable et doit se débarrasser de l'un d'entre eux, c'est-à-dire s'auto-ioniser. L'équilibre entre attraction et répulsion dépend essentiellement de la position relative entre les électrons et le noyau. La position relative moyenne entre les électrons d'un atome est définie par la mécanique quantique. L'atome ne présente donc un excès de répulsion par rapport à l'attraction nucléaire que dans des états quantiques excités bien définis, dits états auto-ionisants (états dont l'énergie est supérieure à l'énergie mini-

M

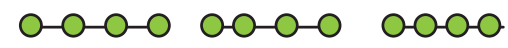
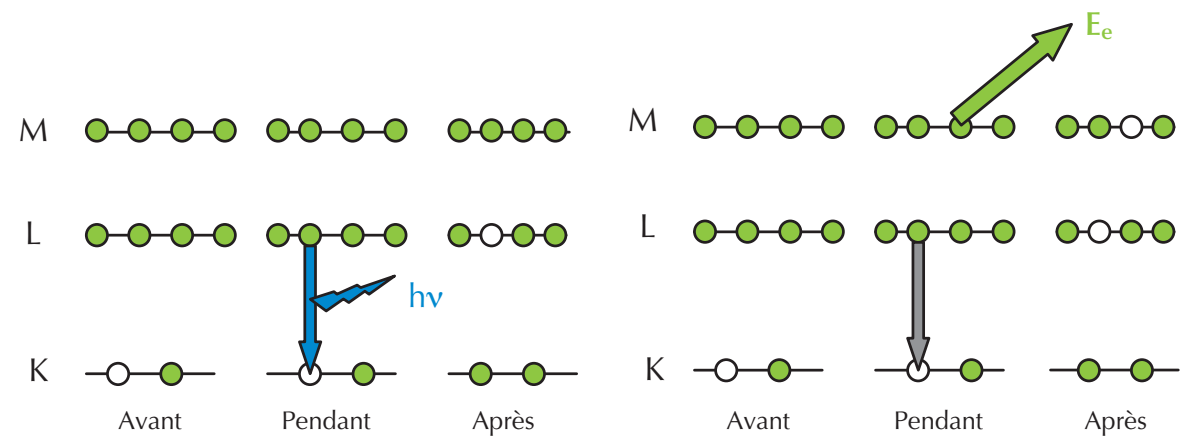

Émission de rayon $\mathrm{X}$ $\mathrm{h} v=\mathrm{B}_{\mathrm{K}}-\mathrm{B}_{\mathrm{L}}$

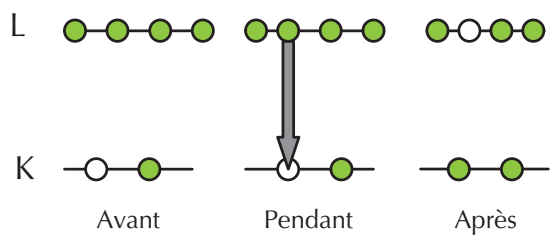

Émission Auger $E_{e} \# B_{K}-B_{L}-B_{M}$

2. Émissions $\mathrm{X}$ et Auger. (En vert : états électroniques occupés ; en blanc : états électroniques vides)

male nécessaire permettant l'éjection d'un électron du cortège).

L'énergie d'un état atomique ionisé dans une couche donnée $\mathrm{X}$ est définie par son potentiel d'ionisation $B_{X}$, énergie nécessaire pour extraire cet électron de l'atome. L'énergie des atomes ionisés croît lorsque l'ionisation provient de couches de plus en plus profondes: $\mathrm{B}_{\mathrm{K}}>\mathrm{B}_{\mathrm{L}}>\ldots>\mathrm{B}_{\mathrm{V}}(\mathrm{V}$ : niveau de valence). Tout atome ionisé en couche interne a reçu une quantité d'énergie supérieure à l'énergie minimale nécessaire permettant l'éjection de l'électron le moins lié du cortège atomique (électron de valence), et se trouve donc dans un état auto-ionisant. Ces atomes ionisés en couche profonde (K par exemple) peuvent se désexciter par deux processus :

- soit par une transition radiative vers un état ionisé en couche L (émission d'un photon $\mathrm{X}$, d'énergie $\mathrm{h} v=\mathrm{B}_{\mathrm{K}}-\mathrm{B}_{\mathrm{L}}$, fig. 2a) ;
- soit par émission spontanée d'un électron quelconque : par exemple, dans le cas de la figure $2 b$ (transition Auger, dite KLM), la lacune en couche $\mathrm{K}$ étant comblée par un électron $\mathrm{L}$, l'énergie disponible $\mathrm{B}_{\mathrm{K}}-\mathrm{B}_{\mathrm{L}}$ est transférée, non radiativement, à un électron $\mathrm{M}$; ce dernier sera spontanément éjecté avec une énergie $\mathrm{E}_{\mathrm{e}} \# \mathrm{~B}_{\mathrm{K}}-\mathrm{B}_{\mathrm{L}}-\mathrm{B}_{\mathrm{M}}$ (\#, car retirer un électron $\mathrm{M}$ d'un atome déjà ionisé en couche L nécessite un peu plus d'énergie que pour un atome neutre).

\section{Les articles fondateurs de 1923 et 1925}

Pierre Auger, fasciné par les expériences de Wilson qui observait dans des chambres à détente les trajectoires formées par des gouttelettes de liquide lors du passage de particules chargées émises au cours de désintégrations nucléaires, décide en 1922 d'étudier avec cet appareil les trajectoires 
des électrons éjectés dans un processus de photo-ionisation. Il construit alors une chambre de Wilson, qu'il modifie pour permettre l'observation d'électrons de faible énergie. Il étudie systématiquement et patiemment avec cette chambre les trajectoires des photo-électrons émis par des atomes ionisés en couche profonde et observe [2] qu'il se passe quelque chose au voisinage de leurs points d'émission. Il découvre après de nombreux essais qu'il y a souvent deux électrons émis et non un (un électron «dur» : le photo-électron, et un électron " mou », de plus faible énergie, que les physiciens appelleront plus tard électron Auger).

On savait qu'un atome ionisé en couche profonde peut se désexciter par émission d'un photon X ; Pierre Auger subodore que l'émission de cet électron "supplémentaire " pourrait être due à un processus alternatif jamais encore observé de désexcitation de l'atome ionisé lors d'une « explosion » se produisant dans son cortège électronique, mais il doit le démontrer. $\mathrm{La}$ probabilité d'émission d'un photon $\mathrm{X}$ varie extrêmement rapidement avec le numéro atomique $Z$ des atomes, suivant une loi en $Z^{4}$. Il n'y avait aucune raison a priori pour qu'il en soit de même en ce qui concerne l'effet qu'il vient de découvrir (on démontrera plus tard que dans l'approximation non relativiste la probabilité d'émission Auger ne varie pas avec Z). Pierre Auger étudie [2] alors l'intensité relative de ces deux processus en fonction du numéro atomique et démontre expérimentalement en 1925, dans un article peu connu mais fondamental, que le rapport de branchement entre les deux processus, le rendement de fluorescence, varie très rapidement avec Z. L'effet Auger constitue le mode dominant de désexcitation des atomes légers ionisés en couche profonde, alors que l'émission X constitue le mode prépondérant de désexcitation des atomes lourds. Pierre Auger avait découvert un processus aussi important en physique que l'émission des rayons X. Il fallait une audace incroyable pour déduire de clichés aussi peu lisibles, à l'aide d'une technique aussi démoniaque, l'existence de l'émission d'un second électron, et encore plus pour en expliquer correctement l'origine. Il lui a aussi fallu certainement une très grande modestie pour accepter qu'une découverte de cette importance reste ignorée si longtemps.

La difficulté expérimentale fondamentale à surmonter pour étudier l'effet Auger est de mesurer la très faible énergie des électrons " mous " émis par des atomes isolés. Lors de leur passage dans la matière où ils sont émis, ces électrons sont ralentis dès leurs premières collisions avec les atomes, dégradant inéluctablement l'information essentielle qu'ils contiennent : leur énergie. Il faut donc que l'atome isolé qui émet un électron Auger se trouve dans un vide poussé et que l'électron émis ne rencontre aucun obstacle avant d'être analysé dans un détecteur. La technique la plus précise pour mesurer l'énergie d'un électron est de le faire passer dans un champ électrique ou magnétique (ou les deux), et d'analyser sa trajectoire ; le détecteur doit ainsi lui aussi se trouver sous un vide poussé. Ces difficultés de nature technologique, principalement liées au vide, telles que la mise au point de sources d'atomes émetteurs d'électrons Auger en jet gazeux et d'enceintes de transfert sous vide différentiel, ne furent résolues que dans les années 1965-70.

Ainsi fut ouverte l'ère de la spectroscopie Auger en tant notamment que technique d'analyse (voir visuel p. 11) et rendant possible de nombreuses découvertes en physique fondamentale [3]. Cette technique représente un marché de plusieurs millions de dollars.

\section{La « redécouverte » de l'effet Auger}

L'effet Auger, oublié ou ignoré des physiciens atomistes, a été "redécouvert » en 1963 par Madden et Codling au National Bureau of Standards (NBS) à Gaithersburg. La problématique était la suivante : dans les atomes d'hélium on observait toutes les transitions radiatives émises par des atomes dont un seul électron était excité, mais aucune transition radiative correspondant à la désexcitation d'atomes dont deux électrons auraient été excités. La question était même posée de savoir si ces états, a priori très difficiles à préparer (on ne disposait pas à cette époque de sources lumineuses possédant assez d'énergie pour photo-exciter doublement les atomes) existaient vraiment.

R. P. Madden et K. Codling [4] ont alors utilisé la première source de lumière présentant un spectre UV continu, dans lequel on pouvait piocher l'énergie désirée pour exciter doublement l'hélium : le premier anneau de rayonnement synchrotron construit au NBS. Ces auteurs ont réalisé une expérience de spectroscopie d'absorp- tion et mis en évidence des raies dont les énergies correspondaient exactement à toutes celles calculées de double excitation de l'hélium : ces états existaient (ils étaient formés puisque des photons étaient absorbés par ces atomes), mais ne se désexcitaient pas par émission radiative.

À la même époque, on commençait à disposer de spectrographes électrostatiques permettant d'étudier les électrons de basse énergie. Madden et Codling ont alors observé que ces états doublement excités se désexcitaient par émission d'électrons, qu'ils ont appelés électrons d'auto-ionisation, et dont les énergies correspondaient exactement... à celles d'électrons Auger ; ceci n'est en fait pas étonnant puisque ces états doublement excités qui possèdent une énergie supérieure à celle du premier potentiel d'ionisation sont des états autoionisants. Quelques années plus tard on observa la désexcitation par émission de photons X de ces états doublement excités, mais avec une probabilité bien inférieure à celle de l'émission d'électrons "Auger". Il y avait donc compétition entre les deux processus ; on "redécouvrait " la notion de rendement de fluorescence, introduite par Pierre Auger en 1925.

\section{Le problème à trois corps}

La photo-ionisation de l'atome d'hydrogène est un processus bien compris : un photon de fréquence $v$ est absorbé par un atome et son électron envoyé dans le continuum avec une énergie hv - B (B : énergie de liaison de l'électron). Dans le cas d'un atome comportant plus d'un électron, l'énergie disponible est cédée à l'ensemble de cet atome, un de ses électrons étant éjecté dans le continuum avec une énergie donnée par la même formule que dans l'hydrogène : hv - B, n'entraînant aucun inconfort intellectuel pour le physicien. Jusqu'en 1967, faute d'informations expérimentales contradictoires, ce modèle s'est imposé allant jusqu'à laisser certains physiciens postuler, comme cela a été écrit dans plusieurs livres de physique, qu'il ne serait pas possible, pour des raisons fondamentales (?), d'extraire plus d'un électron de l'atome avec un photon unique. Ce n'est qu'en 1967, dans une expérience célèbre, mais dérangeante, que Carlson et Krause [5] ont démontré expérimentalement qu'un atome pouvait émettre, après absorption d'un photon, deux électrons se partageant d'une manière continue l'énergie disponible. 
L'effet Auger, tout comme l'ionisation double, est par définition un processus à deux électrons (au moins), donc un problème à trois corps (deux électrons et un noyau). La technique utilisée par les physiciens atomistes pour décrire les atomes à plusieurs électrons, consiste à représenter le problème à n corps comme la composition de $\mathrm{n}$ problèmes à deux corps. Cette technique a conduit, avec un remarquable succès, à la théorie de Hartree qui représente les propriétés de chaque électron dans le champ moyen créé par tous les autres, ignorant l'interaction spécifique de deux électrons individuels entre eux ; mais elle génère de grandes difficultés conceptuelles pour la description des processus à trois corps, qui ont été appelés plus tard " effets de corrélation ». Ces difficultés sont certainement à l'origine du manque d'intérêt de la collectivité des physiciens atomistes pour cet effet essentiel, mais ne peuvent totalement l'expliquer.

L'effet Auger a été le premier effet atomique ne pouvant être réduit à un problème élémentaire à deux corps. Le développement dans les années 60-70 de la spectroscopie Auger a permis, au-delà des applications techniques en physique du solide ou des surfaces, la naissance d'une nouvelle physique et la découverte d'un monde inattendu d'effets nouveaux; ces effets, exclusivement fondés sur l'interaction (répulsion) d'un certain nombre d'électrons d'un même atome entre eux, sont brièvement illustrés ci-dessous.

\section{La physique « post-Auger »}

La première de ces découvertes importantes a été l'observation en 1967, citée ci-dessus, de l'ionisation double des atomes par un photon unique, processus dans lequel l'énergie d'excitation de l'atome est partagée, selon un spectre continu entre deux électrons : un des électrons ayant un spectre piqué vers les hautes énergies, l'autre vers les basses énergies (fig. 3).

Quelques années plus tard, un nouveau processus de désexcitation d'un atome ionisé, conceptuellement assez étrange, mélangeant l'électrodynamique quantique et le simple effet de répulsion coulombienne entre les électrons atomiques, a été découvert par T. Aberg [6] : 1'effet Auger radiatif. Lors de ce processus, de faible probabilité, un atome dans un état excité auto-ionisant se désexcite en émettant simultanément un photon et un électron (fig. 4).

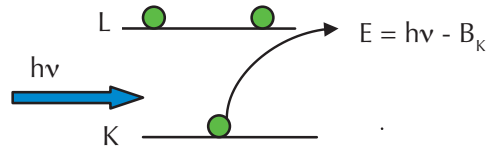

Photo-ionisation simple

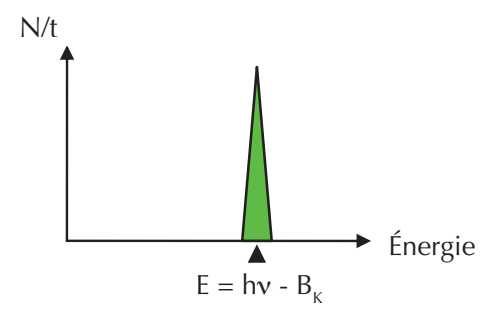

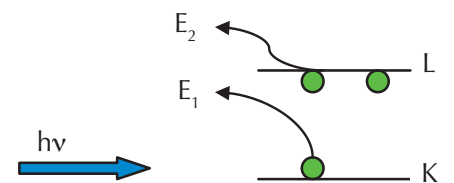

Photo-ionisation double $\mathrm{E}_{1}+\mathrm{E}_{2}=\mathrm{hv}-\mathrm{B}_{\mathrm{K}}-\mathrm{B}_{\mathrm{L}}$

$\mathrm{N} / \mathrm{t}$

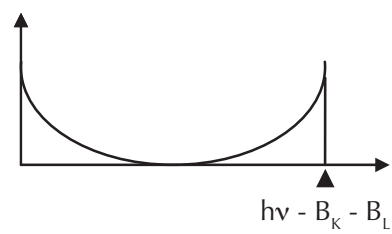

3. Ionisation simple et ionisation double provoquées par absorption d'un photon d'énergie hv. Haut de la figure : schémas de photo-ionisation ; bas de la figure : spectres d'énergie des électrons observés dans les deux cas ( $\mathrm{N} / \mathrm{t}$ : nombre d'électrons émis par unité de temps).

E, $E_{1}, E_{2}$ : énergies des photo-électrons. $B_{K}, B_{L}$ : énergies de liaison des électrons en couches $K$ et $L$.

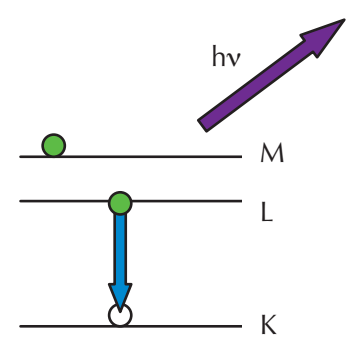

Émission de rayon $\mathrm{X}$

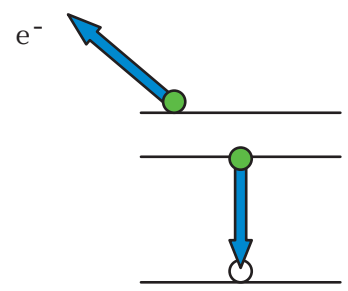

Émission Auger

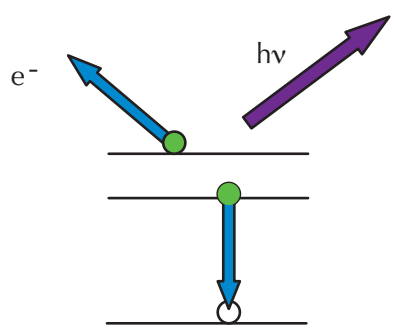

Auger radiatif

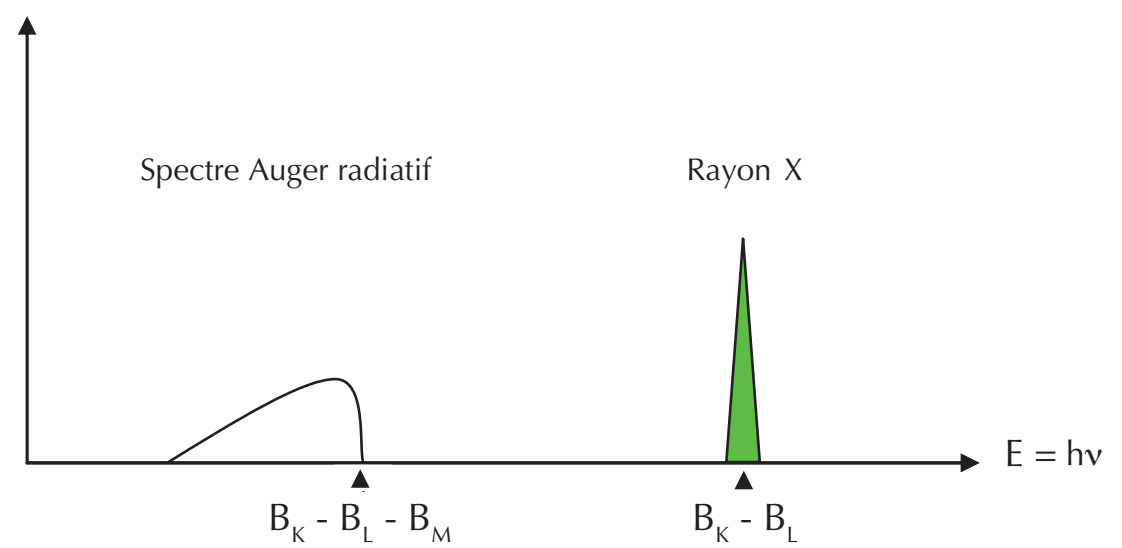

Spectre de rayons $X$ Auger radiatif

4. Effet Auger radiatif d'un atome ionisé en couche K. En haut : schémas de désexcitation de l'atome ionisé. En bas : spectre radiatif observé. À droite, en vert, la raie K correspondant à l'émission X simple (processus le plus probable). À gauche, le spectre radiatif (d'intensité beaucoup plus faible) émis lors d'une transition Auger radiative. L'énergie disponible $\left(B_{K}-B_{k}\right)$ se partage de manière continue entre un électron (non observable dans ce spectre radiatif) et un photon. $\left(B_{K}-B_{L}\right)-B_{M}$ correspond à l'énergie maximale du spectre radiatif continu émis. 
L'état final de la transition est le même que lors d'une émission Auger (l'atome est ionisé une fois de plus qu'avant la transition), mais l'énergie disponible se repartit entre un photon et un électron présentant des spectres continus piqués vers les hautes et basses énergies, d'une manière analogue à ce qui est observé lors d'une ionisation double par un photon unique.

Chaque transition Auger causant une ionisation supplémentaire, la succession rapide en cascade de plusieurs transitions Auger peut conduire à des situations tout à fait extraordinaires. Un atome ionisé en couche profonde, la couche $\mathrm{K}$ par exemple, peut se désexciter par émission Auger (un électron L par exemple comblant la lacune $\mathrm{K}$, un autre étant éjecté dans le continuum) : l'atome dans son état final est alors deux fois ionisé en couche L. Ces états d'ionisation $\mathrm{L}$ peuvent alors de nouveau se désexciter par transitions Auger laissant l'atome encore plus ionisé, et ainsi de suite, générant une cascade d'ionisations spontanées (cascade Auger), ne s'arrêtant que lorsque toutes les lacunes se trouvent en couche externe. On peut ainsi, à l'issue d'une longue cascade de désexcitations Auger, trouver les atomes dans des états extrêmement ionisés, comme l'a montré expérimentalement Nils Perrin, le neveu de Pierre Auger ; le simple processus d'absorption d'un photon (photo-ionisation) peut ainsi conduire à la formation dans un matériau solide ou un tissu humain (radiologie) d'un site extrêmement ionisé produisant des effets durables ou irréversibles.

Une situation encore plus étonnante se produit lors de l'interaction, à très basse vitesse, d'atomes complètement ionisés avec des surfaces. Les ions extrêmement chargés approchant, touchant ou pénétrant une surface, capturent un grand nombre d'électrons jusqu'à neutralisation complète ou même au-delà, mais, pour des raisons énergétiques, dans des états très excités formant des espèces exotiques, dont tous les électrons sont en inversion de population : les atomes creux. Ces atomes, dont les couches profondes sont totalement vides et tous les électrons dans des états extrêmement excités, reviennent à leur état fondamental par de longues cascades de transitions Auger. Ces cascades Auger peuvent se produire librement dans le vide ou, au contraire, dans la matière (sous les surfaces) alterner avec de longues séries de capture.

- Dans le vide, les ions très lents et de numéro atomique $\mathrm{Z}$ élevé, après avoir capturé, au-dessous d'une distance donnée $z_{0}$ de la surface de l'ordre de quelques nm, un certain nombre d'électrons dans des états très excités, sont rétrodiffusés par les charges statiques positives qu'ils ont laissé sur la surface. Les atomes creux ainsi formés se désexcitent alors par une cascade Auger ; ils se re-ionisent donc très rapidement, tout en s'échappant de la zone de capture (au-delà de $z_{0}$ ), c'est-à-dire sans pouvoir se réalimenter en électrons, pour redevenir, dans l'état final, des ions de charge pouvant aller jusqu'à Z-1 (la dernière étape conduisant à un atome simplement excité qui n'est pas auto-ionisant).

- Sous les surfaces, les atomes creux formés dans la matière se désexcitent également par cascades Auger, mais peuvent être réalimentés en électrons dans leurs états excités, pendant, après, ou en alternance avec ces processus d'auto-ionisation. Ces processus de capture peuvent être plus rapides ou plus lents que la cascade Auger en fonction de la charge initiale, de la vitesse de l'ion et de la nature électrique du matériau. Un seul atome peut ainsi changer d'état plus d'une centaine de fois en quelques dizaines de femtosecondes, c'est-à-dire sur des distances qui, en fonction de la vitesse de l'ion, vont de l'ordre de la taille d'un atome jusqu'à quelques dizaines de $\mathrm{nm}$, permettant d'étudier en détail la structure des premières couches d'une surface.

Ces quelques exemples montrent que l'effet découvert il y a près de 90 ans par Pierre Auger n'a certainement pas fini de nous étonner, aussi bien sur le plan conceptuel que sur celui de ses applications.

\section{En savoir plus}

1・ 0 . Hardouin Duparc, Reflets de la physique, 18 (mars 2010) 23-25.

2• P. Auger, Comptes Rendus Acad. Sci. Paris, 177 (1923) 169 et 180 (1925) 65.

3- Le Vide, Les Couches Minces, Numéro spécial consacré à Pierre Auger 50 (1994).

4• R. P. Madden et K. Codling, Phys. Rev. Lett., 10 (1963) 516.

5• M.0. Krause, T. A. Carlson et R.D. Dismuke, Phys. Rev., 170 (1968) 37.

6• T. Aberg et J. Utrianen, Phys. Rev. Lett., 25 (1970) 1745.

Sur la radioactivité et la structure atomique, consulter : - R. D. Evans, Le noyau atomique, Dunod (1961).

- E. U. Condon et G. H. Shortley, The theory of atomic spectra, Cambridge University Press (1935). 